\title{
Impact of Adverse Events on Health Utility and Health-Related Quality of Life in Patients Receiving First-Line Chemotherapy for Metastatic Breast Cancer: Results from the SELECT BC Study
}

\author{
Yasuhiro Hagiwara $^{1}$ (D) Takeru Shiroiwa ${ }^{2} \cdot$ Kojiro Shimozuma $^{3}$ - Takuya Kawahara ${ }^{4}$ \\ Yukari Uemura $^{4}$ - Takanori Watanabe ${ }^{5} \cdot$ Naruto Taira $^{6} \cdot$ Takashi Fukuda $^{2}$. \\ Yasuo Ohashi ${ }^{7} \cdot$ Hirofumi Mukai $^{8}$ \\ Published online: 17 October 2017 \\ (c) The Author(s) 2017. This article is an open access publication
}

\begin{abstract}
Objective The aim of this study was to investigate the impact of adverse events (AEs) on health utility and healthrelated quality of life (HRQOL) in patients with metastatic breast cancer undergoing first-line chemotherapy.

Methods We analyzed the data from the SELECT BC study, a multicenter, open-label, randomized, phase III study conducted in Japan, which compared first-line S-1
\end{abstract}

Electronic supplementary material The online version of this article (doi:10.1007/s40273-017-0580-7) contains supplementary material, which is available to authorized users.

Yasuhiro Hagiwara

hagiwara@epistat.m.u-tokyo.ac.jp

1 Department of Biostatistics, Graduate School of Medicine, The University of Tokyo, 7-3-1 Hongo, Bunkyo-ku, Tokyo 113-0033, Japan

2 Department of Health and Welfare Services, National Institute of Public Health, Wako, Japan

3 Department of Biomedical Sciences, College of Life Sciences, Ritsumeikan University, Kusatsu, Japan

4 Biostatistics Division, Clinical Research Support Center, The University of Tokyo Hospital, Tokyo, Japan

5 Department of Breast Surgery, National Hospital Organization Sendai Medical Center, Sendai, Japan

6 Department of Breast and Endocrine Surgery, Okayama University Hospital, Okayama, Japan

7 Department of Integrated Science and Engineering for Sustainable Society, Faculty of Science and Engineering, Chuo University, Tokyo, Japan

8 Department of Breast and Medical Oncology, National Cancer Center Hospital East, Kashiwa, Japan with taxane therapies. Heath utility and HRQOL were assessed using the EQ-5D-3L and European Organization for Research and Treatment of Cancer Quality of Life Questionnaire Core 30 (EORTC QLQ-C30) at baseline and 3,6 , and 12 months after treatment initiation. Health utility was calculated based on societal preferences, and AEs were reported at each cycle of the study treatment. Linear marginal mean models were used to quantify the impact of the last AEs (with 10 or more incidences) observed before HRQOL assessment on health utility and HRQOL.

Results Analysis included 380 patients and 12 (of 15) AEs. Grade 1 nausea and oral mucositis, grade 1 and 2 edema, and grade 2 fatigue, motor and sensory neuropathy, and myalgia were significantly associated with disutility, measured using the EQ-5D-3L. Grade 1 oral mucositis, grade 1 and 2 fatigue, and grade 2 sensory neuropathy were significantly associated with impaired global health status in the EORTC QLQ-C30. AEs associated with decrements in the five functioning scales included fatigue, oral mucositis, nausea, edema, motor and sensory neuropathy, and myalgia.

Conclusions We reported disutilities caused by AEs in patients with metastatic breast cancer under chemotherapy. These findings can be applied to future model-based costeffectiveness analyses.

Trial Registration Number C000000416. 


\section{Key Points}

Analysis of EQ-5D and adverse event data in a randomized trial revealed that nausea, oral mucositis, edema, fatigue, motor and sensory neuropathy, and myalgia were significantly associated with disutility in patients receiving first-line chemotherapy for metastatic breast cancer.

These disutilities directly measured from patients can be applied to future model-based costeffectiveness analyses for better decision making regarding medical resource allocation.

\section{Introduction}

Breast cancer remains the most common malignancy in women, with an estimated 1.7 million diagnosed cases and 521,900 mortalities worldwide in 2012 [1]. For metastatic breast cancer, the goal of treatment is to prolong survival time and maintain a good quality of life. Although endocrine therapy is standard for certain breast cancer types, chemotherapy is recommended for others, or in endocrine therapy-resistant cases [2]. Chemotherapy effectively prolongs survival time [3, 4] but often induces a variety of adverse events (AEs).

Effective medical resource allocation requires evaluation of not only clinical effectiveness but also cost effectiveness [5]. Model-based analysis plays an important role in assessing the cost effectiveness of chemotherapy drugs, when several sources of data should be combined [6, 7]. In model-based cost-effectiveness analysis, health utility values for each health state are required to calculate quality-adjusted life-years (QALYs) [8]. For example, the remaining life of a metastatic breast cancer patient can be divided into two health states: pre-progression and postprogression states. Health utility values of each state are used as quality weights for the time spent in each state, and disutility caused by AEs are accounted for by subtracting the impact of AEs from the health utility values of each state.

Several researchers have reported the impact of chemotherapy-induced AEs on health utility in metastatic breast cancer patients [9-13]. However, few studies using vignette-based methods, such as the standard gamble and time trade-off methods, obtained health-related quality of life (HRQOL) directly from patients [9, 12]. Some guidelines recommend that HRQOL used in cost-effective analysis be measured directly from patients and then valued based on societal preferences [14-17]. Although one report on the impact of AEs on the EQ-5D-3L index, measured from metastatic breast cancer patients, is available, this study was conducted in a single institution with a small sample size, and the analysis pooled all grades to one category [18]. These limitations led to imprecise and inaccurate estimates. Additional studies for disutility caused by AEs using patient HRQOL data are required.

The aim of this study was to investigate the impact of AEs on health utility, measured using the EQ-5D-3L, in metastatic breast cancer patients receiving first-line chemotherapy. We previously reported the long-term EQ5D-3L index results from the SELECT BC study, a Japanese multicenter, open-label, randomized, phase III study comparing the oral fluoropyrimidine derivative drug S-1 with taxane as first-line chemotherapy for metastatic breast cancer [19]. The results included health utility values before progression, after progression, and immediately before death. In this study, we used the same EQ-5D-3L data as from the SELECT BC study to estimate disutility caused by AEs. To support interpretation, we also reported the impact of AEs on HRQOL, measured by the European Organization for Research and Treatment of Cancer Quality of Life Questionnaire Core 30 (EORTC QLQC30), using the same data.

\section{Methods}

\subsection{Study Population}

We used data from the SELECT BC study, a multicenter, open-label, randomized, phase III, non-inferiority study conducted in Japan, in which oral S-1 was compared with taxane (paclitaxel or docetaxel) as first-line chemotherapy for metastatic breast cancer (registered in the UMIN Clinical Trials Registry as C000000416) [20]. The primary endpoint of this randomized study was overall survival, while the secondary endpoints included safety and HRQOL, which we used in the present study. Women aged 20-75 years with histologically confirmed human epidermal growth factor receptor 2 (HER2)-negative and endocrine therapy-resistant breast cancer, metastatic disease at presentation or recurrence after surgery, an Eastern Cooperative Oncology Group performance status score of $0-1$, and no previous history of chemotherapy for their recurrent or metastatic disease, were eligible for participation in the study. Patients were randomly assigned 1:1 to receive either taxane or $S-1$ therapy. Patients assigned to taxane therapy received one of the following regimens intravenously, as chosen by physicians; docetaxel $60-75 \mathrm{mg} / \mathrm{m}^{2}$ at intervals of 3-4 weeks; paclitaxel $80-100 \mathrm{mg} / \mathrm{m}^{2}$ weekly for 3 of 4 weeks; or paclitaxel $175 \mathrm{mg} / \mathrm{m}^{2}$ at intervals of 3-4 weeks. Patients assigned to $\mathrm{S}-1$ therapy received oral $\mathrm{S}-1$ at a dose of 80,100 , or 
$120 \mathrm{mg} /$ day twice daily, according to their body-surface area, on days 1 through 28 of a 42-day course. The study treatment was continued until tumor progression, occurrence of unacceptable AEs, or completion of six courses (18 or 24 weeks) in the taxane group or four courses (24 weeks) in the S-1 group. Continuation of assigned chemotherapy beyond the specified courses was allowed at the discretion of physicians.

Based on feasibility, HRQOL was assessed in a subset of institutions participating in the SELECT BC study. The collection of high-quality HRQOL data requires cooperation with clinical research coordinators. Institutions in which enough cooperation from clinical research coordinators was unavailable were excluded from HRQOL assessment before initiation of the SELECT BC study.

\subsection{Assessment of Health Utilities, Health-Related Quality of Life (HRQOL), and Adverse Events (AEs)}

We assessed health utility and HRQOL using the EQ-5D3L and EORTC QLQ-C30 questionnaires, respectively $[21,22]$. Responses to the EQ-5D-3L questionnaire were converted into health utility using a validated algorithm based on societal preferences of the Japanese general population [23], considering that the SELECT BC study was conducted in Japan. As reference, we also used the value sets for the UK and the US [24, 25]. Additionally, responses to the EORTC QLQ-C30 questionnaire were converted into a single global health status, five functioning scales, and nine symptom scales using the EORTC-recommended scoring guideline [22]. We used the global health status and five functioning scales as HRQOL in the present study. The EQ-5D-3L and EORTC QLQ-C30 were assessed at baseline and 3, 6, and 12 months after the start of the study treatment. At each point, assessment was conducted before the start of the next treatment cycle. Although the EQ-5D-3L assessment continued beyond 12 months in the SELECT BC study [19], we excluded that data in the present study because a few patients continued the study treatment during that period.

All AEs were continuously assessed in accordance with the Common Terminology Criteria for Adverse Events (CTCAE) version 3.0 during the study treatment [26]. The grade and incidence date of each $\mathrm{AE}$ were reported for every course of the study treatment. Data on the recovery date from AEs was limited, hence we did not use the data.

\subsection{Statistical Analysis}

We analyzed data from the patient population enrolled in the SELECT BC study that completed both the EQ-5D-3L and EORTC QLQ-C30 questionnaires at baseline. Because
AEs were not assessed after the study treatment, we included only the EQ-5D-3L and EORTC QLQ-C30 measured before the end of the last course of the study treatment. We summarized profiles of health utility and HRQOL, and calculated completion rates, defined as the number of completed questionnaires divided by the number of expected responses, excluding patients after the study treatment. We examined predictive factors of incomplete assessments using a multivariable logistic generalized estimating equation.

For processing AE data, we chose 15 non-hematological AEs relevant to the treatment of metastatic breast cancer: febrile neutropenia, fever, fatigue, alopecia, allergy, diarrhea, oral mucositis, nausea, vomiting, anorexia, edema, motor neuropathy, sensory neuropathy, arthralgia, and myalgia. We then identified the last grade of each $\mathrm{AE}$ immediately before EQ-5D-3L and EORTC QLQ-C30 assessment (i.e. 3, 6, and 12 months). We counted the total number of incidences for each $\mathrm{AE}$ at three time points, and calculated the difference between incidence date of AEs and assessment date of the EQ-5D-3L and EORTC QLQC30. Finally, we selected AEs with ten or more incidences for subsequent analysis, meaning that we did not analyze AEs with fewer incidences because those estimates that essentially reflect the small number of assessments would be unstable and imprecise.

We used linear marginal mean models with time-dependent AEs to quantify the impact of each AE on health utility and HRQOL [27]. Analyses using the linear marginal mean model were adjusted for baseline scores, age, treatment, time, and treatment-by-time interaction (see Online Resource 1 for more details) [28]. First, we conducted separate analysis, where only a single analyzable AE (i.e. ten or more incidences) was included in the model. We then conducted simultaneous analysis, where all analyzable AEs were included in the models. Grades of AEs were modeled using linear and quadratic terms. We fitted the models using the generalized estimating equation method [27].

All analyses were conducted using SAS version 9.4 (SAS Institute Inc., Cary, NC, USA). All $p$ value evaluations were two-sided, and a $p$ value $<0.05$ was considered nominally statistically significant without multiplicity adjustment.

\section{Results}

\subsection{Patient Characteristics}

Of 618 randomized patients, 405 were included in the QOL population. After excluding 25 patients with incomplete baseline EQ-5D-3L or EORTC QLQ-C30 questionnaires, 
Table 1 Baseline characteristics in the analysis population

\begin{tabular}{lcc}
\hline & Taxane group $(n=174)$ & S-1 group $(n=206)$ \\
\hline Age [median (IQR)] & $57(50-64)$ & $59(53-65)$ \\
Hormone receptor status & & \\
ER-positive, PR-positive, or both & $126(72.4)$ & $147(71.4)$ \\
ER-negative and PR-negative & $45(25.9)$ & $53(25.7)$ \\
Unknown & $3(1.7)$ & $6(2.9)$ \\
HER2 status & & $190(92.2)$ \\
Negative & $162(93.1)$ & $16(7.8)$ \\
Unknown & $12(6.9)$ & $22(10.7)$ \\
Treatment history & & $61(29.6)$ \\
Oral fluoropyrimidine & $26(14.9)$ & $111(53.9)$ \\
Taxane & $48(27.6)$ & \\
Endocrine therapy & $99(56.9)$ & $44(19.9)$ \\
Disease-free interval & & $66(32.0)$ \\
$<2$ years & $34(19.5)$ & $67(32.5)$ \\
2-5 years & $51(29.3)$ & $32(15.5)$ \\
$\geq 5$ years & $58(33.3)$ & $77(37.4)$ \\
No surgery & $31(17.8)$ & $129(62.6)$ \\
Liver metastasis & $60(34.5)$ & \\
Yes & $114(65.5)$ & \\
No & &
\end{tabular}

Data are expressed as $n(\%)$ unless otherwise specified

$I Q R$ interquartile range, $E R$ estrogen receptor, $P R$ progesterone receptor, HER2 human epidermal growth factor receptor 2 the analysis population consisted of 380 patients (174 in the taxane group and 206 in the $\mathrm{S}-1$ group). Table 1 shows the baseline characteristics of the analysis population. When the two groups were pooled, median age was 58 years (interquartile range $51-65$ ), $72 \%$ of patients had positive hormone receptor status, and $36 \%$ of patients had liver metastasis at baseline.

\subsection{Completion Rates and Profiles of Health Utility and HRQOL}

Table 2 shows the completion rates and profiles of health utility measured using the EQ-5D-3L, and the global health status in the EORTC QLQ-C30. Completion rates were more than $85 \%$ at all three assessment points. Odds ratios for incomplete EQ-5D-3L assessments were $1.17[95 \%$ confidence interval (CI) 0.94-1.46] for the last health utility (per 0.1 points) before incompletion, 1.30 (95\% CI 0.94-1.78) for age (per 10 years), and 1.23 (95\% CI $0.62-2.45)$ for the S-1 group. No AE was significantly associated with incomplete EQ-5D-3L assessments. At baseline, health utility and global health status had similar distributions between the two groups. Thereafter, the S-1 group had a better trend of health utility and global health status than the taxane group. Mean health utility ranged from 0.753 to 0.784 in the taxane group, and 0.806 to 0.848 in the $\mathrm{S}-1$ group.

\subsection{Incidence of AEs}

Of the 15 relevant AEs, the following 12 had ten or more incidences: fatigue, alopecia, diarrhea, oral mucositis, nausea, vomiting, anorexia, edema, motor neuropathy, sensory neuropathy, arthralgia, and myalgia (Table 3). An incidence rate of more than $10 \%$ was observed for fatigue, alopecia, diarrhea, nausea, anorexia, edema, and sensory neuropathy. For all AEs, grades 3 or higher were rare. The median interval between the incidence of AEs and assessment of health utility and HRQOL ranged from 21 to 28 days.

\subsection{Impact of AEs on Health Utility and HRQOL}

Figure 1 and eTable 1 (Online Resource 1) show the impact of AEs on health utility measured by the EQ-5D-3L using societal preferences in Japan. On separate analysis, a significant negative impact was observed for all analyzable AEs, except for anorexia and diarrhea (eTable 1). However, in the simultaneous analysis, a significant negative impact was only observed for fatigue, oral mucositis, nausea, edema, motor neuropathy, sensory neuropathy, and 
Table 2 Completion and profiles of health utility measured using the EQ-5D-3L, and global health status using the EORTC QLQ-C30

\begin{tabular}{|c|c|c|c|c|c|c|}
\hline & \multicolumn{3}{|l|}{ Taxane group } & \multicolumn{3}{|l|}{ S-1 group } \\
\hline & Expected response & Completed response (\%) & Mean (SD) & Expected response & Completed response (\%) & Mean (SD) \\
\hline \multicolumn{7}{|c|}{ Health utility, months } \\
\hline 0 & 174 & $174(100.0)$ & $0.766(0.156)$ & 206 & $206(100.0)$ & $0.768(0.166)$ \\
\hline 3 & 151 & $137(90.7)$ & $0.773(0.153)$ & 167 & $145(86.8)$ & $0.813(0.160)$ \\
\hline 6 & 95 & $86(90.5)$ & $0.753(0.160)$ & 119 & $105(88.2)$ & $0.806(0.166)$ \\
\hline 12 & 33 & $29(87.9)$ & $0.784(0.184)$ & 67 & $60(89.6)$ & $0.848(0.159)$ \\
\hline \multicolumn{7}{|c|}{ Global health status, months } \\
\hline 0 & 174 & $174(100.0)$ & $61.2(22.4)$ & 206 & $206(100.0)$ & $59.3(22.0)$ \\
\hline 3 & 151 & $140(92.7)$ & $62.0(22.7)$ & 167 & $145(86.8)$ & $64.7(20.3)$ \\
\hline 6 & 95 & $86(90.5)$ & $58.1(23.7)$ & 119 & $105(88.2)$ & $64.0(24.2)$ \\
\hline 12 & 33 & $29(87.9)$ & $59.5(23.5)$ & 67 & $59(88.1)$ & $64.5(24.2)$ \\
\hline
\end{tabular}

The number of expected responses at each assessment point was calculated by subtracting the number of patients after the end of the study treatment from the number of patients included in the present study

EORTC QLQ-C30 European Organization for Research and Treatment of Cancer Quality of Life Questionnaire Core 30, SD standard deviation

Table 3 Incidence of adverse events immediately before three assessment points of health utility and HRQOL

\begin{tabular}{lllllll}
\hline & Grade 0 & Grade 1 & Grade 2 & Grade 3 & Grade 4 & Interval, days [median (IQR) $^{\mathrm{a}}$ \\
\hline Febrile neutropenia & $565(99.3)$ & - & - & $4(0.7)$ & $0(0.0)$ & $23(10-41)$ \\
Fever & $567(99.6)$ & $1(0.2)$ & $1(0.2)$ & $0(0.0)$ & $0(0.0)$ & $23(3-42)$ \\
Fatigue & $402(70.7)$ & $150(26.4)$ & $15(2.6)$ & $2(0.4)$ & $0(0.0)$ & $27(19-36)$ \\
Alopecia & $364(64.0)$ & $63(11.1)$ & $142(25.0)$ & - & - & $21(15-28)$ \\
Allergy & $563(98.9)$ & $5(0.9)$ & $1(0.2)$ & $0(0.0)$ & $0(0.0)$ & $21(21-35)$ \\
Diarrhea & $511(89.8)$ & $46(8.1)$ & $12(2.1)$ & $0(0.0)$ & $0(0.0)$ & $21(14-33)$ \\
Oral mucositis & $521(91.6)$ & $45(7.9)$ & $3(0.5)$ & $0(0.0)$ & $0(0.0)$ & $25(15-41)$ \\
Nausea & $511(89.8)$ & $52(9.1)$ & $6(1.1)$ & $0(0.0)$ & $0(0.0)$ & $28(20-40)$ \\
Vomiting & $559(98.2)$ & $9(1.6)$ & $1(0.2)$ & $0(0.0)$ & $0(0.0)$ & $28(17-35)$ \\
Anorexia & $468(82.2)$ & $92(16.2)$ & $9(1.6)$ & $0(0.0)$ & $0(0.0)$ & $28(19-41)$ \\
Edema & $497(87.3)$ & $50(8.8)$ & $21(3.7)$ & $1(0.2)$ & $0(0.0)$ & $24(21-28)$ \\
Motor neuropathy & $556(97.7)$ & $12(2.1)$ & $1(0.2)$ & $0(0.0)$ & $0(0.0)$ & $21(14-28)$ \\
Sensory neuropathy & $433(76.1)$ & $107(18.8)$ & $27(4.7)$ & $2(0.4)$ & $0(0.0)$ & $24(18-28)$ \\
Arthralgia & $519(91.2)$ & $47(8.3)$ & $3(0.5)$ & $0(0.0)$ & $0(0.0)$ & $21(21-28)$ \\
Myalgia & $534(93.8)$ & $29(5.1)$ & $6(1.1)$ & $0(0.0)$ & $0(0.0)$ & $21(18-28)$ \\
\hline
\end{tabular}

Data are expressed as $n(\%)$ unless otherwise specified

Percentage was calculated as the number of incidences divided by the number of total assessments of EQ-5D-3L or EORTC QLQ-C30 (569 assessments) at three assessment points. Multiple incidences in one patient were counted separately

EORTC QLQ-C3O European Organization for Research and Treatment of Cancer Quality of Life Questionnaire Core 30, HRQOL health-related quality of life, $I Q R$ interquartile range

${ }^{a}$ Interval between incidence date of grade 1 or higher adverse events and assessment date of health utility and HRQOL

myalgia (Fig. 1). More than 0.1-point decrements in health utility were observed for grade 2 motor neuropathy and grade 2 myalgia in the simultaneous analysis. Similar results were also obtained based on societal preferences in the UK and the US (eTable 3 in Online Resource 1).

Figure 2 and eTable 2 (Online Resource 1) show the impact of AEs on global health status in the EORTC QLQ-C30. In the separate analysis, a significant negative impact was observed for fatigue, oral mucositis, anorexia, and sensory neuropathy (eTable 2). Of these, only fatigue, oral mucositis, and sensory neuropathy were significantly associated with impaired scores in the simultaneous analysis (Fig. 2). No AE was associated with more than 10-point decrements in global health status in the simultaneous analysis. 


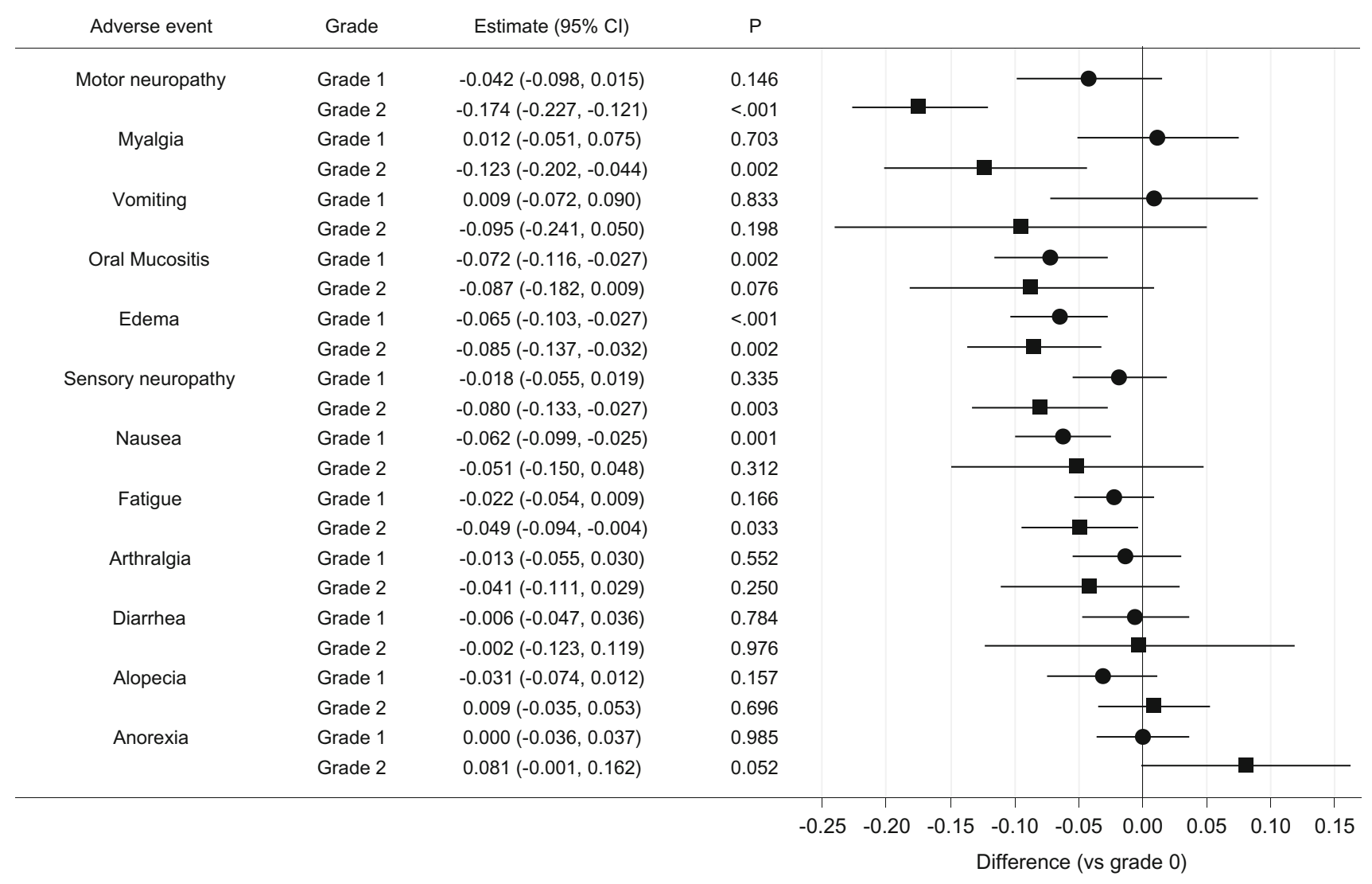

Fig. 1 Impact of adverse events on health utility measured by the EQ-5D-3L in the simultaneous analysis. Estimates comparing grade 1 or 2 with grade 0 by simultaneous analysis based on societal

In simultaneous analyses of the five functioning scales in the EORTC QLQ-C30, significant impairment was observed in grade 2 motor neuropathy $(-21.0 ; 95 \%$ CI -25.4 to -16.7 ) and grade 2 sensory neuropathy $(-6.9 ; 95 \%$ CI -12.3 to -1.5$)$ for physical functioning; grade 1 motor neuropathy $(-14.2 ; 95 \%$ CI -26.3 to -2.0 ), grade 1 and 2 fatigue $(-6.7$ and $-6.8 ; 95 \% \mathrm{CI}$ -10.6 to -2.9 , and -12.3 to -1.4 , respectively), and grade 2 myalgia $(-19.9 ; 95 \% \mathrm{CI}-36.8$ to -3.1$)$ for role functioning; grade 1 fatigue $(-4.0 ; 95 \%$ CI -7.4 to $-0.6)$ and grade 1 edema $(-4.8 ; 95 \% \mathrm{CI}-9.3$ to -0.2$)$ for emotional functioning; grade 1 oral mucositis $(-6.2$; $95 \% \mathrm{CI}-12.1$ to -0.2$)$ for cognitive functioning; grade 1 nausea $(-5.9 ; 95 \% \mathrm{CI}-11.4$ to -0.5$)$ and grade 1 edema $(-5.6 ; 95 \% \mathrm{CI}-11.1$ to -0.1$)$ for social functioning.

\section{Discussion}

In the present study, we investigated the impact of AEs on health utility and HRQOL in metastatic breast cancer patients, using the EQ-5D-3L and EORTC QLQ-C30 data from the SELECT BC study. The results suggest that preferences in Japan are displayed. Adverse events are ranked from top to bottom based on the grade 2 estimates. $C I$ confidence interval

fatigue, oral mucositis, nausea, edema, motor neuropathy, sensory neuropathy, and myalgia were associated with significant disutility, and that fatigue, oral mucositis, and sensory neuropathy were significantly associated with impaired global health status in the EORTC QLQ-C30. These findings can contribute to enhancing model-based cost-effectiveness analysis of chemotherapy by providing disutility values caused by AEs in analysis models.

To the best of our knowledge, there are no comprehensive reports regarding the impact of grade 1 or 2 AEs on the health utility of metastatic breast cancer patients undergoing chemotherapy. Although direct comparison is not feasible due to the difference in grades and populations, some of our findings are consistent with results from previous studies on grade 3 or 4 AEs which used vignettebased methods [9-13]. For example, grade 3 or 4 oral mucositis was reportedly associated with health utility decrements of 0.151 [11], and disutility caused by grade 1 and 2 oral mucositis was 0.072 and 0.087 in the present study, respectively. Severe neuropathy (of unspecified grade) was reportedly associated with health utility decrements of 0.22 units [10], and disutility caused by grade 2 motor and sensory neuropathy was 0.174 and 0.080 in the 


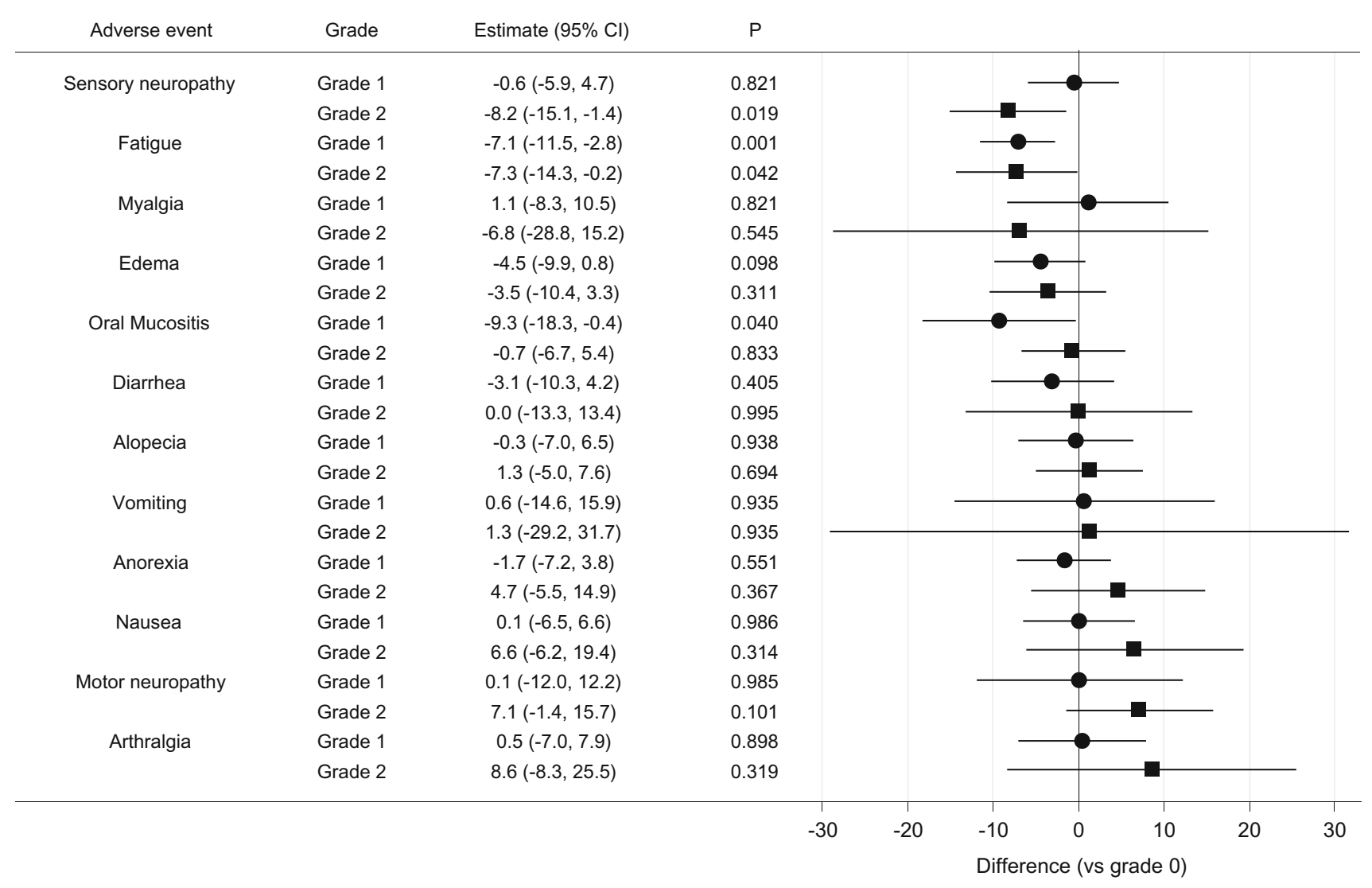

Fig. 2 Impact of adverse events on global health status in the EORTC QLC-C30 in the simultaneous analysis. Estimates comparing grade 1 or 2 with grade 0 by simultaneous analysis are displayed. Adverse events are ranked from top to bottom based on the grade 2

present study, respectively. However, inconsistencies were also observed between the previous and present studies. For example, alopecia (of unspecified grade) was reportedly associated with health utility decrements of 0.114 [11], whereas disutility caused by grade 1 and 2 alopecia was below 0.05 and not significant in the present study (note that alopecia only has grades of $0-2$ in CTCAE version 3.0). Grade 3 or 4 diarrhea was reportedly associated with health utility decrements of 0.29 and 0.19 [13], whereas disutility caused by grade 1 and 2 diarrhea was approximately 0 and not significant in the present study. The difference in the latter $\mathrm{AE}$ may be explained by the interval between the incidence of AEs and the assessment of HRQOL (see below for more discussion). When one applies estimates of disutility caused by AEs in the present study to other cancers, caution is advised because it is unclear whether these estimates are generalizable to other diseases. Specifically, all patients included in our analyses were female. If available, disease-specific disutility estimates would be better.

Our findings reveal that different AEs affected different functioning scales in the EORTC QLQ-C30. Although estimates. CI confidence interval, EORTC QLQ-C30 European Organization for Research and Treatment of Cancer Quality of Life Questionnaire Core 30

only three of seven AEs with a significant negative impact on health utility were associated with decrements in global health status, all seven AEs had a significant negative impact on at least one functioning scale. In addition, all AEs that had a significant negative impact on at least one functioning scale were detected as AEs that significantly affected health utility. This supports the multiattribute system for health utility assessment that has been adopted in the EQ-5D in assessing the impact of AEs induced by chemotherapy for metastatic breast cancer. Although the EQ-5D does not directly measure symptoms related to AEs, our findings suggest that the EQ-5D could capture these symptoms indirectly by measuring their effects on functioning in daily life. Recently, mapping studies from the EORTC QLQ-C30 to the EQ-5D have been actively conducted [29-31]. Because the cancer-specific EORTC QLQ-C30 including the nine symptom scales is considered to be sensitive to chemotherapy-induced AEs, the extent of disutility may be different between direct EQ-5D index values and mapped EQ-5D index values from the EORTC QLQ-C30, and is worthy of further investigation. 
In the present study, we used data from a randomized controlled trial to assess the impact of AEs on health utility. This novel study design has several advantages relative to previous study designs. First, HRQOL is measured directly from cancer patients undergoing treatment, a recommended procedure in some countries [14-17]. Second, a large dataset is available due to longitudinal HRQOL assessment (562 EQ-5D index values were used in the present study). Third, assessment of AEs is standardized by the CTCAE. Standardization of description for AEs is one of the challenges in vignette-based methods [32].

However, this study design also has disadvantages. First, severe AEs cannot be assessed because the incidence of such events is usually low in randomized controlled trials under close monitoring with appropriate dose adjustment. Second, the impact of temporary AEs such as nausea and diarrhea may have been under-evaluated because they have often disappeared at assessment of HRQOL, conducted at the start of the next treatment cycle. To assess grade 3 or 4 AEs or temporary AEs, other study designs that do not use randomized trial data would be appropriate [32, 33]; the proxy version of the EQ-5D [14, 34], which has been applied in other research fields such as dementia [35], may be a feasible option worth investigating. Third, settings in randomized trials can be different from those in routine care. Eligibility criteria, strict treatment regimens, blinding, written informed consent, and intensive management in randomized trials may affect the association of AEs with health utility and HRQOL.

In addition to the above, other limitations should be considered when interpreting our study findings. First, incomplete assessment of health utility and HRQOL cannot be avoided in randomized controlled trials. From the observed data, we cannot eliminate the possibility that poor health status was associated with incomplete assessment and led to attenuated association of AEs with health utility and HRQOL. However, completion rates through three assessment points were so high that it would be unlikely that the results were substantially biased. Second, we could not assess the cumulative and interactive effects of AEs. Some AEs such as neuropathy and edema have a persistent nature, but time spent with these AEs could not be modeled due to modeling complexities relative to sample size. In addition, some chemotherapy drugs often induce multiple AEs simultaneously, and this can be accounted for by modeling the interaction between multiple AEs. However, in general, statistical power for detecting interaction would be low in a study similar in size to the present study. Instead, our analysis, which assumed no interaction in additive scale [36], provided stable estimates by averaging potential interaction. Third, we did not account for comorbidity in our analyses due to the lack of data. Elderly patients with cancer often have comorbid conditions [37], which may affect both the incidence of AEs and health utility.

\section{Conclusions}

We investigated the impact of AEs on the health utility of metastatic breast cancer patients undergoing first-line chemotherapy, using the SELECT BC study data. Fatigue, oral mucositis, nausea, edema, motor neuropathy, sensory neuropathy, and myalgia were significantly associated with disutility, measured using the EQ-5D-3L. These results were supported by the cancer-specific EORTC QLQ-C30 results. Incorporating disutility caused by AEs into cost-effectiveness models would result in more accurate QALY estimates and lead to better decision making in allocating medical resources.

Acknowledgements This study was supported by the Comprehensive Support Project for Health Outcomes Research (CSP-HOR) of the Public Health Research Foundation. We are grateful to the SELECT BC study investigators, staff, and patients who participated.

Author contributions Conception and design: YH, TS, KS, TK, TW, TF, YO, and HM. Collection and assembly of data: TW, NT, and HM. Statistical analysis: YH, TK, YU, and YO. Interpretation: YH, TS, KS, and TK. Manuscript preparation: YH. Manuscript review: YH, TS, KS, TK, YU, TW, NT, TF, YO, and HM.

\section{Compliance with Ethical Standards}

Funding The SELECT BC study was sponsored by the Comprehensive Support Project for Oncology Research (CSPOR) of the Public Health Research Foundation. The research fund was provided to CSPOR by Taiho Pharmaceutical Co., Ltd. under the study contract.

Conflict of interest Yasuhiro Hagiwara was contractually employed by EP CRSU, and Yasuo Ohashi received consulting fees or honoraria from the Public Health Research Foundation and Taiho Pharmaceutical Co., Ltd. Takeru Shiroiwa, Kojiro Shimozuma, Takuya Kawahara, Yukari Uemura, Takanori Watanabe, Naruto Taira, Takashi Fukuda, and Hirofumi Mukai have declared no conflicts of interest.

Ethical approval The SELECT BC study was conducted in accordance with the Declaration of Helsinki and the Ethical Guidelines for Clinical Research of the Japanese Ministry of Health, Labor and Welfare. The study protocol was approved by an independent Ethics Committee for each participating site. All participants provided written informed consent.

Data availability statement The datasets generated and/or analyzed during the current study are available from the corresponding author on reasonable request.

Open Access This article is distributed under the terms of the Creative Commons Attribution-NonCommercial 4.0 International License (http://creativecommons.org/licenses/by-nc/4.0/), which permits any noncommercial use, distribution, and reproduction in any medium, provided you give appropriate credit to the original author(s) and the source, provide a link to the Creative Commons license, and indicate if changes were made. 


\section{References}

1. Torre LA, Bray F, Siegel RL, et al. Global cancer statistics, 2012. CA Cancer J Clin. 2015;65:87-108.

2. Partridge AH, Rumble RB, Carey LA, et al. Chemotherapy and targeted therapy for women with human epidermal growth factor receptor 2-negative (or unknown) advanced breast cancer: American Society of Clinical Oncology Clinical Practice Guideline. J Clin Oncol. 2014;32:3307-29.

3. Paterson AHG, Szafran O, Cornish F, et al. Effect of chemotherapy on survival in metastatic breast cancer. Breast Cancer Res Treat. 1981;1:357-63.

4. O'Shaughnessy J. Extending survival with chemotherapy in metastatic breast cancer. Oncologist. 2005;10(Suppl 3):20-9.

5. Shih YC, Halpern MT. Economic evaluations of medical care interventions for cancer patients: how, why, and what does it mean? CA Cancer J Clin. 2008;58:231-44.

6. Tappenden P, Chilcott J, Ward S, et al. Methodological issues in the economic analysis of cancer treatments. Eur $\mathrm{J}$ Cancer. 2006;42:2867-75.

7. Diaby V, Tawk R, Sanogo V, et al. A review of systematic reviews of the cost-effectiveness of hormone therapy, chemotherapy, and targeted therapy for breast cancer. Breast Cancer Res Treat. 2015;151:27-40.

8. Drummond MF, Sculpher MJ, Torrance GW, O'Brien BJ, Stoddart GL. Cost-utility Analysis. In: Drummond MF, Sculpher MJ, Torrance GW, O'Brien BJ, Stoddart GL, editors. Methods for the economic evaluation of health care programmes. 3rd ed. Oxford: Oxford University Press; 2005.

9. Leung PP, Tannock IF, Oza AM, Puodziunas A, Dranitsaris G. Cost-utility analysis of chemotherapy using paclitaxel, docetaxel, or vinorelbine for patients with anthracycline-resistant breast cancer. J Clin Oncol. 1999;17:3082-90.

10. Brown RE, Hutton J, Burrell A. Cost effectiveness of treatment options in advanced breast cancer in the UK. Pharmacoeconomics. 2001;19:1091-102.

11. Lloyd A, Nafees B, Narewska J, Dewilde S, Watkins J. Health state utilities for metastatic breast cancer. $\mathrm{Br} \mathrm{J}$ Cancer. 2006;95:683-90.

12. Grunberg SM, Weeks J, Magnan WF, et al. Determination of utility scores for control of chemotherapy-induced nausea or vomiting - CALGB 309801. J Support Oncol. 2009;7:W17-20.

13. Frederix GW, Quadri N, Hövels AM. Utility and work productivity data for economic evaluation of breast cancer therapies in the Netherlands and Sweden. Clin Ther. 2013;35:e1-7.

14. National Institute for Health and Clinical Excellence. Guide to the methods of technology appraisal 2013. https://www.nice.org.uk/ guidance/pmg9/resources/guide-to-the-methods-of-technologyappraisal-2013-pdf-2007975843781. Accessed 5 Jun 2017.

15. Tosh JC, Longworth LJ, George E. Utility values in National Institute for Health and Clinical Excellence (NICE) technology appraisals. Value Health. 2011;14:102-9.

16. Fukuda T, Shiroiwa T, Ikeda S, et al. Guideline for economic evaluation of healthcare technologies in Japan (in Japanese). J Natl Inst Public Health. 2013;62:625-40.

17. Shiroiwa T, Fukuda T, Ikeda S, Takura T, Moriwaki K. Development of an official guideline for the economic evaluation of drugs/medical devices in Japan. Value Health. 2017;20:372-8.

18. Tachi $\mathrm{T}$, Teramachi $\mathrm{H}$, Tanaka $\mathrm{K}$, et al. The impact of outpatient chemotherapy-related adverse events on the quality of life of breast cancer patients. PLoS One. 2015;10:e0124169.

19. Shiroiwa T, Fukuda T, Shimozuma K, et al. Long-term health status as measured by EQ-5D among patients with metastatic breast cancer: comparison of first-line oral S-1 and taxane therapies in the randomized phase III SELECT BC trial. Qual Life Res. 2017;26:445-53.

20. Takashima T, Mukai H, Hara F, et al. Taxanes versus S-1 as the first-line chemotherapy for metastatic breast cancer (SELECT $\mathrm{BC}$ ): an open-label, non-inferiority, randomised phase 3 trial. Lancet Oncol. 2016;17:90-8.

21. Brooks R. EuroQol: the current state of play. Health Policy. 1996;37:53-72.

22. Aaronson NK, Ahmedzai S, Bergman B, et al. The European Organization for Research and Treatment of Cancer QLQ-C30: a quality-of-life instrument for use in international clinical trials in oncology. J Natl Cancer Inst. 1993;85:365-76.

23. Tsuchiya A, Ikeda S, Ikegami N, et al. Estimating an EQ-5D population value set: the case of Japan. Health Econ. 2002;11:341-53.

24. Dolan P. Modeling valuations for EuroQol health states. Med Care. 1997;35:1095-108.

25. Shaw JW, Johnson JA, Coons SJ. US valuation of the EQ-5D health states: development and testing of the D1 valuation model. Med Care. 2005;43:203-20.

26. National Cancer Institute. Common terminology criteria for adverse events v3.0 (CTCAE). https://ctep.cancer.gov/ protocoldevelopment/electronic_applications/docs/ctcaev3.pdf. Accessed 5 Jun 2017.

27. Diggle PJ, Heagerty P, Liang KY, Zeger SL. Time-dependent Covariates. In: Fitzmaurice GM, Laird NM, Ware JH, editors. Analysis of longitudinal data. 2nd ed. New York: Oxford University Press; 2002. p. 245-81.

28. Fitzmaurice GM, Laird NM, Ware JM. Marginal models: introduction and overview. In: Fitzmaurice GM, Laird NM, Ware JH, editors. Applied longitudinal analysis. 2nd ed. Hoboken: Willey; 2011. p. 341-52.

29. Kim EJ, Ko SK, Kang HY. Mapping the cancer-specific EORTC QLQ-C30 and EORTC QLQ-BR23 to the generic EQ-5D in metastatic breast cancer patients. Qual Life Res. 2012;21:1193-203.

30. Longworth L, Yang Y, Mulhern B, et al. Use of generic and condition-specific measures of health related quality of life in NICE decision making: a systematic review, statistical modeling and survey. Health Technol Assess. 2014;18:1-224.

31. Doble B, Lorgelly P. Mapping the EORTC QLQ-C30 onto the EQ-5D-3L: assessing the external validity of existing mapping algorithms. Qual Life Res. 2016;25:891-911.

32. Shabaruddin FH, Chen LC, Elliott RA, Payne K. A systematic review of utility values for chemotherapy-related adverse events. Pharmacoeconomics. 2013;31:277-88.

33. Prosser LA, Grosse SD, Wittenberg E. Health utility elicitation: is there still a role for direct methods? Pharmacoeconomics. 2012;30:83-6.

34. Schwenkglenks M, Matter-Walstra K. Is the EQ-5D suitable for use in oncology? An overview of the literature and recent developments. Expert Rev Pharmacoecon Outcomes Res. 2016;16:207-19.

35. Orgeta V, Edwards RT, Hounsome B, et al. The use of the EQ-5D as a measure of health-related quality of life in people with dementia and their carers. Qual Life Res. 2015;24:315-24.

36. Shiroiwa T, Fukuda T, Tsutani K. Health utility scores of colorectal cancer based on societal preference in Japan. Qual Life Res. 2009;18:1095-103.

37. Yancik R, Havlik RJ, Wesley MN, et al. Cancer and comorbidity in older patients: a descriptive profile. Ann Epidemiol. 1996;6:399-412. 